\title{
Study of Lau fringes generated by a photorefractive volume grating
}

\author{
Gustavo Forte ${ }^{\mathrm{a}, \mathrm{c}, *}$, Myrian Tebaldi ${ }^{\mathrm{a}, \mathrm{b}}$, Nestor Bolognini ${ }^{\mathrm{a}, \mathrm{b}, \mathrm{c}}$ \\ a Centro de Investigaciones Ópticas, CIOp (CONICET La Plata-CIC-UNLP), P.O. Box 3, 1897 Gonnet, La Plata, Argentina \\ b UIDET OPTIMO, Departamento de Ciencias Básicas, Facultad de Ingeniería, Universidad Nacional de La Plata, Argentina \\ c Departamento de Física, Facultad de Ciencias Exactas, Universidad Nacional de La Plata, Argentina
}

\section{A R T I C L E I N F O}

\section{Keywords:}

Lau effect

Photorefractive crystal

Volume grating

\begin{abstract}
A B S T R A C T
In this work the Lau fringes generated by using a combination of an amplitude grating and a photorefractive volume phase grating is theoretically and experimentally analyzed. A model based on the path integral formalism to calculate the patterns intensity is employed. We show that the Lau pattern behavior is governed by the output pupil diameter of the imaging recording system, the DC external electric field and the crystal thickness. The introduction of a phase modulation that gathers the previously mentioned parameters allows determining the condition to optimize the fringe visibility. In this case, the visibility maintains a sinusoidal dependence as it happened with planar grating experiments. The experimental results confirm the theoretical model proposed.
\end{abstract}

\section{Introduction}

The Lau effect takes place when an extended light source which is imaged onto a grating illuminates a second identical grating [1]. High contrast fringe patterns appear at infinity only for determined grating separations that is a function of the wavelength of the optical field and the grating period. In order to obtain Lau fringes, the experimental arrangement has been implemented by using an amplitude or a phase second grating [2-4].

The Lau phenomenon has been exploited for implementing optical interferometers $[5,6]$. Lau interferometers are shearing interferometer which have several applications. For example, in the zero distance Lau interferometer, the fringes provide information about the phase structure of a test object under study [7]. In order to quantitatively reconstruct the phase information codified in Lau interferometric fringe patterns a phase stepping technique could be used. The phase steps required by this technique is done with in-plane shift of one of the interferometer gratings [8]. Note that the Lau interferometry has a better signal to noise ratio in comparison with Talbot interferometry $[9,10]$. However, Lau interferometric fringes exhibits in general a low visibility. Then, it is important to find strategies to obtain high contrast Lau fringes.

Lau fringes theoretical model has been done in different frameworks. From the scalar diffraction theory point of view, the intensity distribution at the observation plane can be calculated as the correlation of the intensity distribution of the first amplitude grating with the modulus square of the Fresnel transform of the second grating [11]. In
Ref. [12,13], the effect is explained based on coherence theory. In particular, Sudol et at. demonstrated that there is a fringe dependence on the relationship between the grating separation and the periodicity of the complex degree of spatial coherence in the plane of the second grating.

By using a phase grating with a square-wave modulation as a second grating, Lau patterns visibility varies periodically with the grating separation and its phase modulation [14]. In this particular case, the highest contrast Lau fringes is observed for a distance between the gratings $z_{L}=\left(m+\frac{1}{2}\right) d^{2} / \lambda(m=1,2,3, \ldots)$, where $d$ is the grating period and $\lambda$ the wavelength of the optical field.

The mentioned works were involved with Lau patterns generated by planar gratings. On the other hand, it should be emphasized that fringes generated by a volume grating stored in a photorefractive crystal presents new features [15]. The system behaves as a combination of two amplitude gratings when an analyzer is used at the crystal output. There, the theoretical proposal was focused on the polarization behavior of this photorefractive grating to control the patterns visibility.

In a previous work concerning Talbot patterns, we used a pathintegral approach to explain the self-images features when a volume grating is used instead of a planar grating [16]. It's worth mentioning that the self-images' visibility depends on the volume grating thickness and on the grating modulation. In Ref. [17], the path-integral proposal was extended to determine the self-image patterns' intensity generated by a volume phase grating stored in a BSO crystal. A distinctive result

\footnotetext{
* Corresponding author.

E-mail address: gforte@ciop.unlp.edu.ar (G. Forte).
} 
was that the self-image visibility is enhanced by changing the parameters which characterize the volume phase grating.

In the present paper, we analyze the essential features of the Lau effect by using a photorefractive volume phase grating. In Section 2 the experimental write-in set up is described. A 3D light intensity distribution is calculated by using the optical transfer function (OTF) of the incoherent imaging system. This distribution is compared with experimental results. The photorefractive index grating modulation by 3D light intensity distribution is simulated emphasizing the dependence on the recording parameters. The Lau effect formation by using this volume phase grating is analyzed in Section 3 based on a path integral approach. Also, the theoretical and experimental fringe distributions are presented. The quality of these patterns is evaluated by using the visibility. An adequate phase parameter to analyze the fringes visibility is employed. Finally, our analysis allows to enhance and to control the fringe visibility to be used in interferometric applications.

\section{Volume grating stored in a photorefractive crystal}

\section{1. $3 D$ light intensity distribution}

Let us introduce the photorefractive write-in process in Fig. 1. An amplitude grating, $\mathrm{G}$, with a low frequency square-wave modulation is illuminated by a white light source, $\mathrm{S}_{1}$ through a condenser lens $\mathrm{L}_{1}$. An image of this grating is projected inside a BSO crystal with unitary magnification by means of lens $\mathrm{L}_{2}$. Due to the limitation of the incoherent imaging system frequency behavior, a $0.5 \mathrm{~mm}$ grating period is used. A filter, $\mathrm{F}_{\mathrm{G}}$, centered at wavelength $\lambda_{w}=534 \mathrm{~nm}$ $\left(\Delta \lambda_{w}= \pm 6 \mathrm{~nm}\right)$ is employed in this step due to the strong BSO photoconductivity at this wavelength. An iris diaphragm $\mathrm{P}$ is located behind lens $\mathrm{L}_{2}$ to control the exit pupil. At a distance $z_{i}$ from lens $\mathrm{L}_{2}$ an image of the grating is formed. We are concerned in the light distribution at the image plane and its neighborhood, where $z_{a}$ is a variable distance (see Fig. 1).

By following the same approach as detailed in Ref. [17], the 3D light intensity distribution results

$I\left(z_{0}, x_{o} ; D\right)=\mathrm{F}^{-1}\{\tilde{t}(u) . \mathrm{H}(u)\}$

where $u$ is the variable in the frequency domain associated to the spatial variable $x_{0}$ and $D$ is the output pupil aperture diameter. In Eq. (1) the longitudinal spatial coordinate is $z_{0}=z_{a}-z_{i}$, which is referred to the image plane, $\tilde{t}(u)$ is the Fourier transform of the grating amplitude transmittance function and $\mathrm{H}(u)$ is the OTF corresponding to the

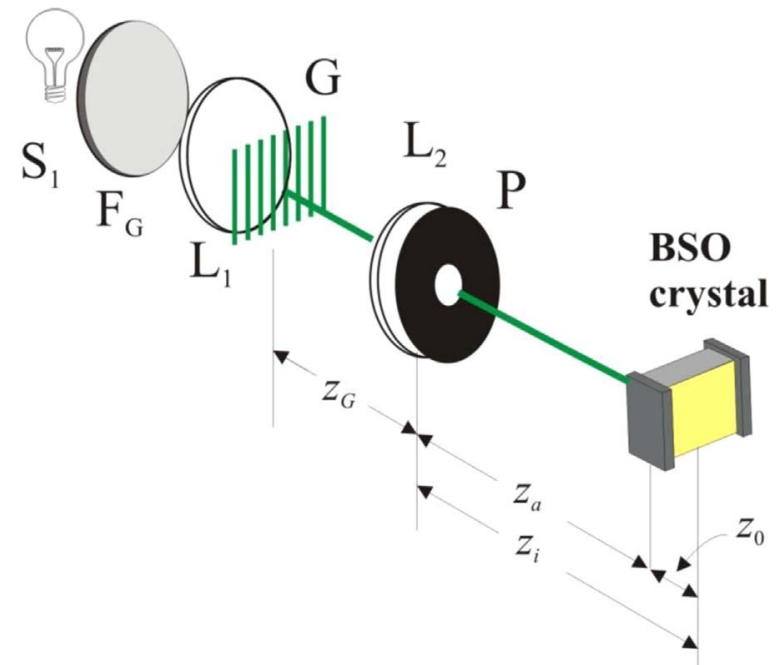

Fig. 1. Write-in experimental set-up: $S_{1}$ : white light source; $F_{G}$ : green interference filter; $\mathrm{L}_{1}$ : condenser lens; $\mathrm{L}_{2}$ : imaging lens of focal length $f$; G: Ronchi grating;; P: diaphragm. incoherent imaging system.

The 3D light intensity distribution in terms of the output pupil is depicted in Fig. 2. Experimental results were measured by replacing in the arrangement of Fig. 1 the BSO crystal by a CCD Pulnix TM-6CN camera. An iris diaphragm gives the variable output pupil diameter, $D$. The $z_{0}$ position of the camera was incremented by steps of $\Delta z_{0}=0.1 \mathrm{~mm}$ from $z_{0}=-3-5 \mathrm{~mm}$. The result of the $z_{0}$-scanning is a stack of Ronchi grating defocused images and the focused image constituting a 3D light intensity distribution. Once these images are found, they were stored in order to obtain their profiles. A 3D graph is generated by putting all together the intensity profiles. Fig. 2(a), (b) and (c) show the experimental 3D profiles. The simulated light intensity distributions in Fig. 2(d), (e) and (f), are calculated from Eq. (1).

The result of Fig. 2(a) shows that the critical defocus of the 3D image is related with a large output pupil aperture diameter $(D=50 \mathrm{~mm})$. Note that the 3D image contrast gradual attenuation and the image contrast reversal are both produced away from the plane $z_{0}=0$. A decreasing $D$ (Fig. 2(b) and (c)) produces an increasing depth of focus. In particular, Fig. 2(c) corresponding to $D=3 \mathrm{~mm}$ shows a lower severity of defocus of the 3D image in comparison with the results of Fig. 2(a) and 2(b). The same behavior of the light intensity distributions is observed in the simulated graphs of Fig. 2(d), (e) and (f).

\subsection{Photorefractive volume grating}

When an optical field is incident into a photorefractive crystal, it induces a space-charge electric field. This electric field generates an index grating through the linear Pockels effect. The index modulation amplitude in BSO crystal results $\Delta n_{0}=-(1 / 2) n_{0}{ }^{3} r_{41} R e\left\{E_{l}\right\}$, where $n_{0}$ is the bulk refractive index, $r_{41}$ is the electro-optic coefficient and $\operatorname{Re}\left\{E_{1}\right\}$ is the real part of the space-charge electric field complex amplitude [18]. In our experimental arrangement, the grating period is $0.5 \mathrm{~mm}$, therefore, the diffusion charge transport mechanism is negligible and only the drift must be considered. In this case, the space-charge electric field can be enhanced by applying a DC external electric field $E_{0}$. It implies that the incident intensity distribution and the photorefractive volume grating are in phase, and therefore $\operatorname{Re}\left\{E_{1}\right\} \approx-E_{0}$. As a consequence, $\Delta n_{0}$ is proportional to $E_{0}$ and it takes values in the order of $10^{-4}-10^{-5}$. The index grating results [17],

$$
\begin{aligned}
n\left(z_{0}, x_{\mathbf{0}} ; E_{0}, D, L_{Z}\right) & =n_{0}+\Delta n\left(z_{0}, x_{0} ; E_{0}, D, L_{Z}\right) \\
& =n_{0}+\Delta n_{0}\left(E_{0}\right) I_{N}\left(z_{0}, x_{0}, D\right) \operatorname{rect}\left(z_{0} / L_{Z}\right)
\end{aligned}
$$

where the normalized 3D light intensity distribution $I_{N}\left(z_{0}, x_{0}, D\right)$ is obtained by Eq. (1) and the rect function limits the grating to the crystal thickness $L_{Z}$. Then, the intensity distribution replicates itself as a modulation of birefringence in the crystal volume.

In Eq. (2) it is observed that the photorefractive volume grating is characterized by using three parameters. One parameter is associated to the imaging system, that is the output pupil diameter, and the other parameters related to the photorefractive register are the external DC electric field and the crystal thickness. The defocusing of the projected image is governed by changing the output pupil diameter. In this way, the region in which the index grating spreads inside the crystal is modified. Besides, the index grating amplitude depending on the spacecharge field is controlled by varying the DC electric field and the volume grating is limited, as maximum, by the crystal thickness.

In Fig. 3, the simulated index gratings evaluated from Eq. (2) are displayed. In order to understand the role playing by the mentioned parameters, one of them is varied and the other parameters are maintained fixed. The BSO constant values used in the calculation are $n_{0}=2.54$ at $\lambda_{w}$ and $r_{41}=3.4 \times 10^{-12} \mathrm{~m} / \mathrm{V}[19,20]$. In Fig. 3(a), (b) and (c) the index grating modulations for crystal thicknesses of $L_{Z}=2,6$ and $10 \mathrm{~mm}, D=50 \mathrm{~mm}$ and $E_{0}=7 \mathrm{kV} / \mathrm{cm}$ are depicted. The serious defocus of the $3 \mathrm{D}$ image that maps the photorefractive grating is related with a 
(a)

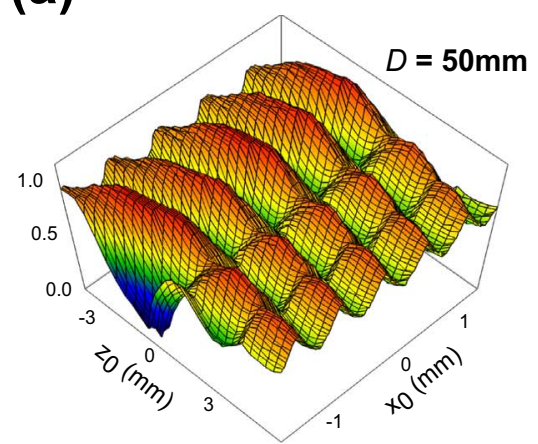

(d)

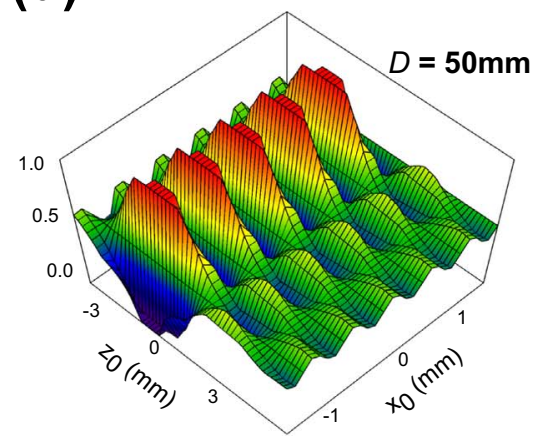

(b)

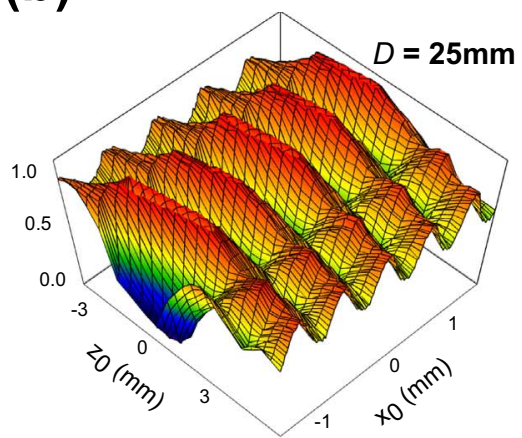

(e)

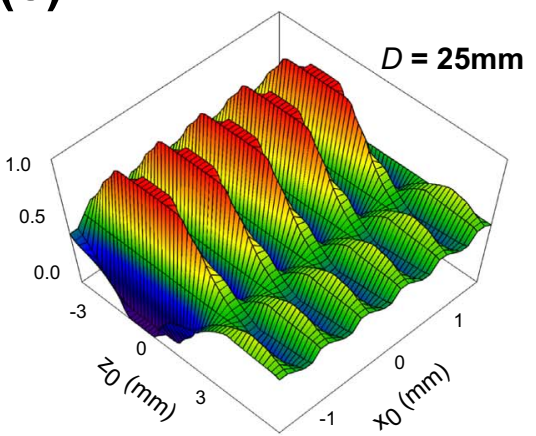

(c)

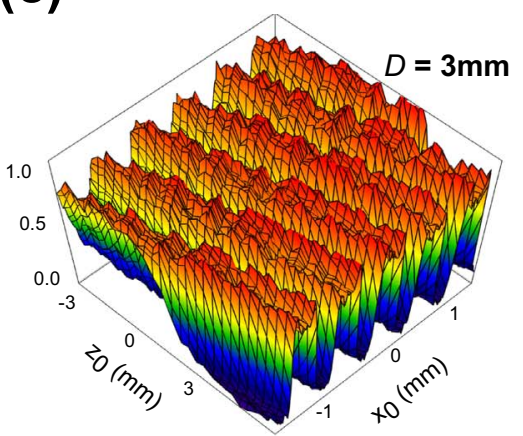

(f)

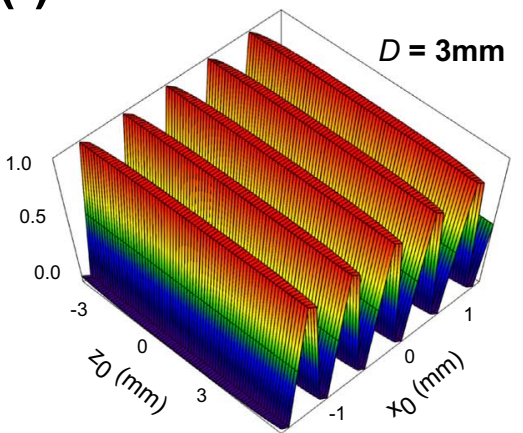

Fig. 2. Experimental (a), (b), (c) and theoretical (d), (e), (f) normalized 3D light intensity distribution for different output pupil diameters, $D$.

large $D$ supported by a large $L_{Z}$. This behavior is evident in the case of $L_{\mathrm{Z}}=6 \mathrm{~mm}$ and $L_{\mathrm{Z}}=10 \mathrm{~mm}$ (see Fig. $3(\mathrm{~b})$ and (c)). It should be pointed out that the attenuation and the reversal of the phase modulation contrast is observed away from the plane $z_{0}=0$.

The results of Fig. 3(d), (e) and (f) correspond to $E_{0}=5,7$ and $9 \mathrm{kV} /$ $\mathrm{cm}, D=3 \mathrm{~mm}$ and $L_{\mathrm{Z}}=6 \mathrm{~mm}$. They show an increasing index grating (a)

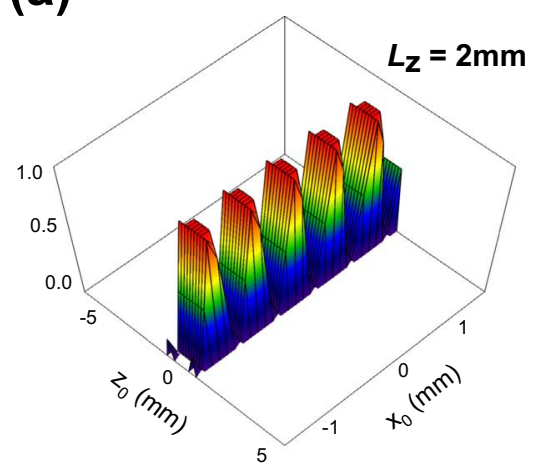

d)

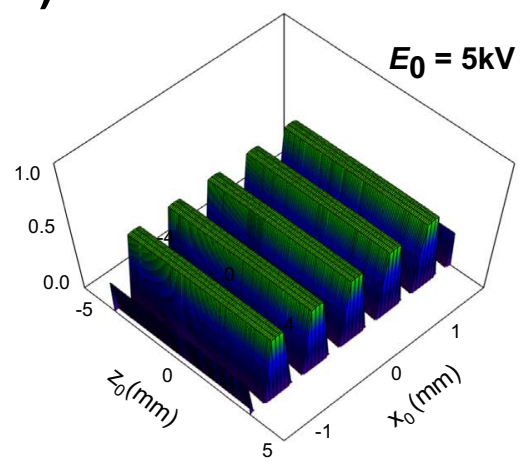

(b)

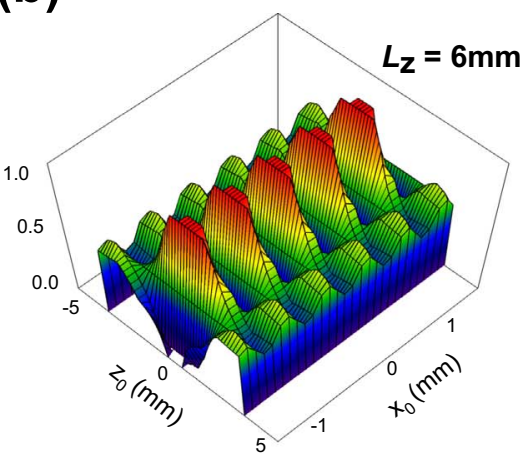

e)

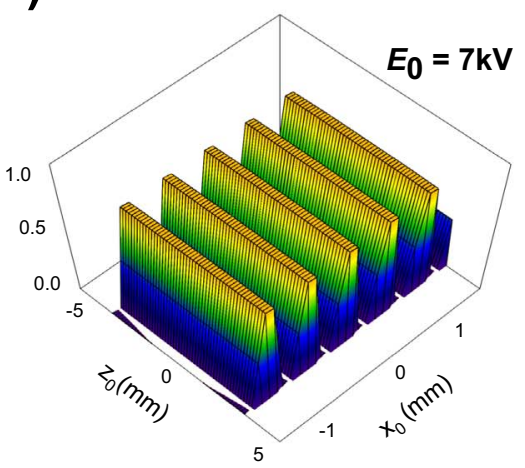

(c)

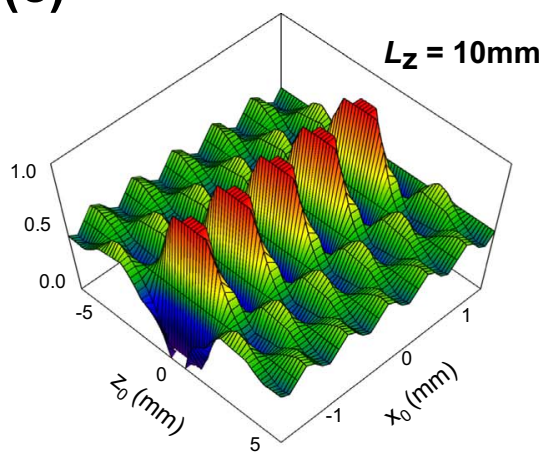

f)

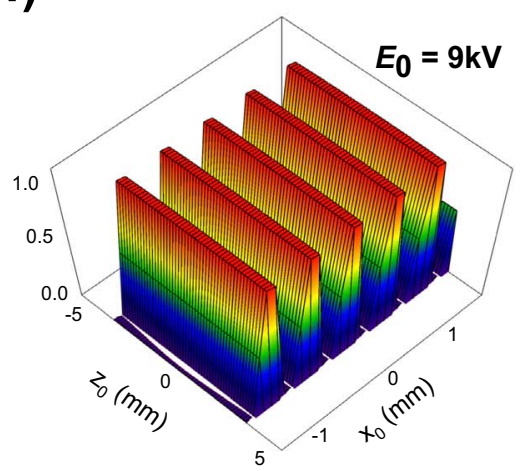

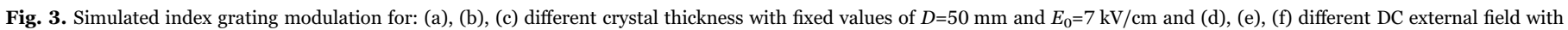
fixed values $D=3 \mathrm{~mm}$ and $L_{Z}=6 \mathrm{~mm}$. 


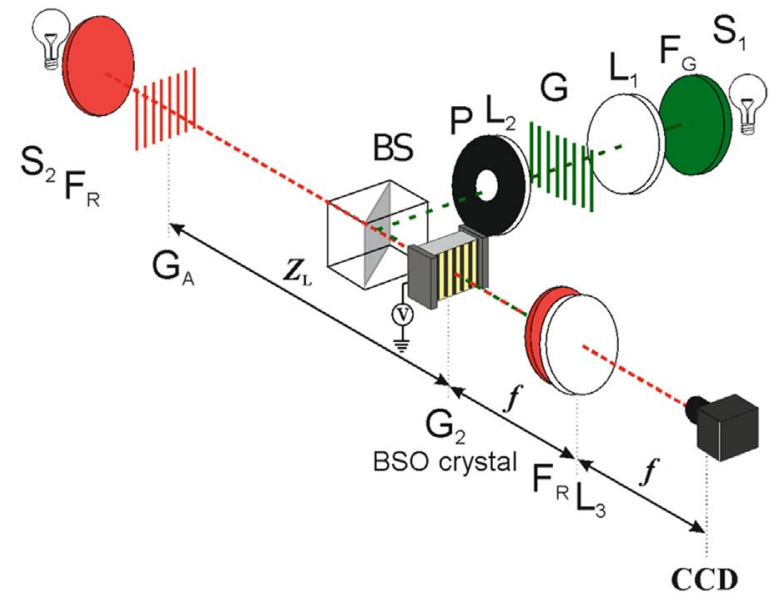

Fig. 4. Lau fringes generation experimental set-up: $S_{1}, S_{2}$ : white light source; $F_{G}, F_{R}$ : green and red interference filter, respectively; $L_{1}, L_{2}, L_{3}$ : lenses; $G$ and $G_{A}$ : amplitude Ronchi gratings;; BS: beam splitter; P: diaphragm; $\mathrm{G}_{2}$ : photorefractive volume phase grating.

modulation amplitude, $\Delta n_{0}$. Note that for a value of $D=3 \mathrm{~mm}$, the region in which the $3 \mathrm{D}$ image spreads is larger than the crystal thickness (even for the larger $L_{Z}=10 \mathrm{~mm}$ ) but the grating is effectively limited by the crystal.

\section{Lau effect by the photorefractive volume grating}

\subsection{Lau patterns formation}

The read-out experimental arrangement is shown in Fig. 4. An incoherent red light $\left(\lambda_{r}=633 \mathrm{~nm}\right)$ illuminates an amplitude grating, $\mathrm{G}_{\mathrm{A}}$, free propagates a distance $z_{L}$ and impinges onto the photorefractive volume grating, $\mathrm{G}_{2}$. The emerging light distribution is Fourier transformed by lens $\mathrm{L}_{3}$, resulting in Lau patterns at the CCD plane.

Lau images intensity is interpreted as the correlation of the intensity distribution of the first amplitude grating with the modulus square of the Fresnel pattern generated by the second grating. In our case, the second grating is a photorefractive volume phase grating. Thus, the intensity distribution at the observation plane is calculated as

$I(x)=|t(x)|^{2} \otimes|\Psi(z, x)|^{2}$

where $\Psi(z, x)$ is the Fresnel pattern generated by the photorefractive volume grating, whose calculation is outlined in the following subsection.

\subsection{Fresnel pattern generated by the photorefractive grating}

The inhomogeneous field propagation through the volume grating can be treated by means of a path integral formulation to obtain the intensity of the Fresnel patterns generated by the photorefractive medium [17]. A straightforward calculation leads to determine the field at the output plane $(z, x, y)$

$$
\begin{aligned}
\Psi(z, x, y)= & \frac{t_{F} k_{r}}{2 \pi i\left(z-L_{Z}\right)} \int_{-\infty}^{\infty} \int_{-\infty}^{\infty} e^{i k_{r}\left[\left(z-L_{Z}\right)+\frac{\left(x-x_{0}\right)^{2}+\left(y-y_{0}\right)^{2}}{2\left(z-L_{Z}\right)}\right]} \\
& e^{i k_{r} \int_{0}^{L Z} n\left(z^{\prime}, x_{0}, y_{0}\right) d z^{\prime}} \Psi\left(0, x_{0}, y_{0}\right) d x_{0} d y_{0}
\end{aligned}
$$

where $\Psi\left(0, x_{0}, y_{0}\right)$ stands for the field just behind the input plane, $n\left(z^{\prime}, x_{0}, y_{0}\right)$ is the spatially variable refractive index $k_{r}=2 \pi / \lambda_{r}$ is the vacuum wave number in the read-out process, $L_{Z}$ is the crystal thickness and $t_{F}$ is the Fresnel transmission coefficient which is approximately constant by assuming a low modulation in the refractive index. Note that in our experimental case a one dimensional grating is considered, therefore, in the following calculation only the $x$ spatial coordinates is taken into account.

Fresnel images generated by the photorefractive volume grating are calculated by means of Eqs. (2) and (4). High contrast Lau fringes are found by evaluating $z=z_{L}=\left(m+\frac{1}{2}\right) d^{2} / \lambda$ in Eq. (3), where $m$ is an integer number and $d$ is the grating period. Then, the behavior of Lau patterns in terms of the previously proposed volume grating parameters will be analyzed. To this purpose, let us consider the visibility. The conventional expression of the visibility is

$V=\frac{\left.|| \Psi\left(z_{L}, 0\right)\right|^{2}-\left|\Psi\left(z_{L}, d / 2\right)\right|^{2} \mid}{\left|\Psi\left(z_{L}, 0\right)\right|^{2}+\left|\Psi\left(z_{L}, d / 2\right)\right|^{2}}$

When using a second planar phase grating with a square-wave modulation, Lau patterns visibility depends on the grating separation, $z$, and phase grating modulation, $\beta$, as

$V=4 \sum_{\substack{n=\text { odd } \\ \text { integer }}}\left|C_{n}\right|^{2} \sin (\beta) \sin \left(\pi \lambda z n^{2} / d^{2}\right)$

where $C_{n}$ is the Fourier coefficient of the square-wave function [14].

In order to analyze our experimental proposal, we consider the replacement of the second planar phase grating in the Lau effect by a photorefractive volume phase grating. Let us suppose that the visibility expressed by Eq. (6) maintains its validity, as the parameter $\beta$ is adequately modified. In a previous paper [17], we proposed a new phase parameter, $\beta_{\text {mod }}$, which takes into account the variable modulation through the volume. This parameter is given by

$\beta_{\text {mod }}=k_{r}\left(\int_{0}^{L_{Z}}\left(\Delta n\left(z^{\prime}, 0 ; E_{0}, D, L_{Z}\right)-\Delta n\left(z^{\prime}, d / 2 ; E_{0}, D, L_{Z}\right)\right) d z^{\prime}\right)$

Note that $\beta_{\text {mod }}$ gathers the dependence on the output pupil diameter of the imaging system, the crystal thickness and the external applied field, which in fact control the features of the index volume phase grating.

Fig. 5(a) and (b) show experimental Lau images produced by photorefractive volume phase gratings generated with crystal thicknesses $L_{\mathrm{Z}}=3$ and $6 \mathrm{~mm}$, respectively. In this case, the remaining parameters are set at $D=3 \mathrm{~mm}$ and $E_{0}=7 \mathrm{kV} / \mathrm{cm}$. It is apparent an increasing fringes contrast for $L_{Z}=6 \mathrm{~mm}$ in comparison with $L_{Z}=3 \mathrm{~mm}$. This fact leads to expect an increasing of the visibility calculated by using Eq. (5). This behavior motivates a deeper analysis of the phenomenon.

Then, the fringes contrast is explored for Lau patterns generated by a photorefractive grating. Fig. 6 depicts the Lau patterns behavior when the phase grating modulation varies in terms of the parameters detailed in Section 2.2. Theoretical results are obtained using Eqs. (2), (3) and (4). The simulated pattern profiles by using index grating modulations corresponding to the crystal thickness $L_{Z}=2,6$ and $10 \mathrm{~mm}$, whereas maintained fixed $D=50 \mathrm{~mm}$ and $E_{0}=7 \mathrm{kV} / \mathrm{cm}$, are presented in Fig. 6(a). A remarkable enhancement of the Lau image visibility is apparent up to $L_{Z}=6 \mathrm{~mm}$. For crystal thickness larger than $6 \mathrm{~mm}$ the Lau pattern visibility decreases. This behavior can be explained by considering the result shown in Fig. 3(c). In this case, a phase modulation contrast reversal of the index grating is observed. The effect of the field propagation through this grating induces a shifting in the Lau fringes reducing thereby the whole fringes visibility. The experimental patterns stacked in Fig. 6(b) confirm this behavior.

Fig. 6(c) shows the behavior of Lau patterns calculated using the same values of $L_{\mathrm{Z}}$ as in Fig. 6(a) but, in this case, for $D=3 \mathrm{~mm}$ and $E_{0}=7 \mathrm{kV} / \mathrm{cm}$. Recalling the result depicted in Fig. 3(e), a single grating is generated along the crystal thickness. Then, a remarkable enhancement of the patterns visibility is observed as a consequence of the accumulated in-phase field propagation through this volume grating. However, the pattern corresponding to $L_{\mathrm{Z}}=10 \mathrm{~mm}$ exhibits a diminishing quality, a fact that is not expected a priori from a grating with $D=3 \mathrm{~mm}$. This behavior will be discussed in Fig. 7. It is observed that the experimental results of Fig. 6(d) verify the theoretical simulation of 


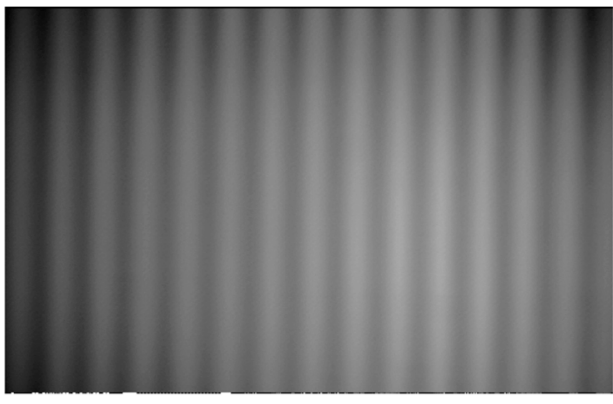

(a)

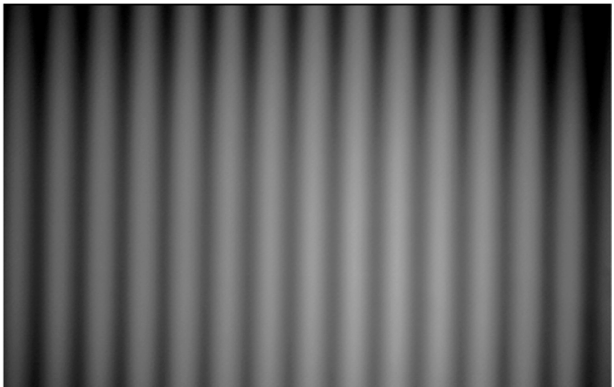

(b)

Fig. 5. Experimental Lau patterns produced by photorefractive gratings with $D=3 \mathrm{~mm}, E_{0}=7 \mathrm{kV} / \mathrm{cm}$, (a) $L_{\mathrm{Z}}=3 \mathrm{~mm}$ and (b) $L_{\mathrm{Z}}=6 \mathrm{~mm}$.

Fig. 6(c).

Fig. 6(e) shows Lau pattern profiles calculated using gratings with $D=3 \mathrm{~mm}, L_{Z}=6 \mathrm{~mm}$ and $E_{0}=5,7$ and $9 \mathrm{kV} / \mathrm{cm}$. In this case, the improvement of the patterns is due to the field propagation through a grating that experiences increasing modulation amplitude expressed by Eq. (2). This behavior is confirmed by the experimental patterns in Fig. 6(f).

Let us analyze quantitatively the visibility of the Lau patterns generated by the photorefractive volume gratings. The pattern visibility defined by Eq. (5) is studied in terms of the phase parameter $\beta_{\text {mod }}$ introduced in Eq. (7). In Fig. 7(a) the visibility of theoretical patterns is plotted in terms of the $\beta_{\text {mod }}$ calculated for the respective volume phase gratings. A sinusoidal fitting curve for the least squares fit confirms the expected visibility behavior. In Fig. 7(b) the visibility of experimental patterns in terms of $\beta_{\text {mod }}$ is considered. A sine function for comparisons is added to the graph.


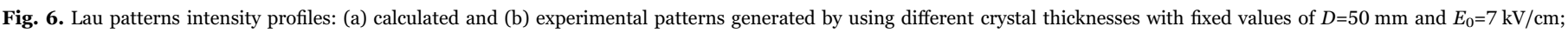

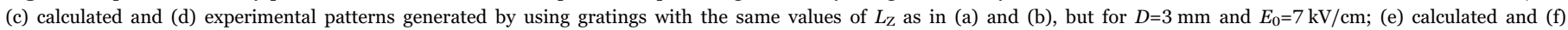
experimental patterns generated by using different DC external field with fixed values $D=3 \mathrm{~mm}$ and $L_{Z}=6 \mathrm{~mm}$. 

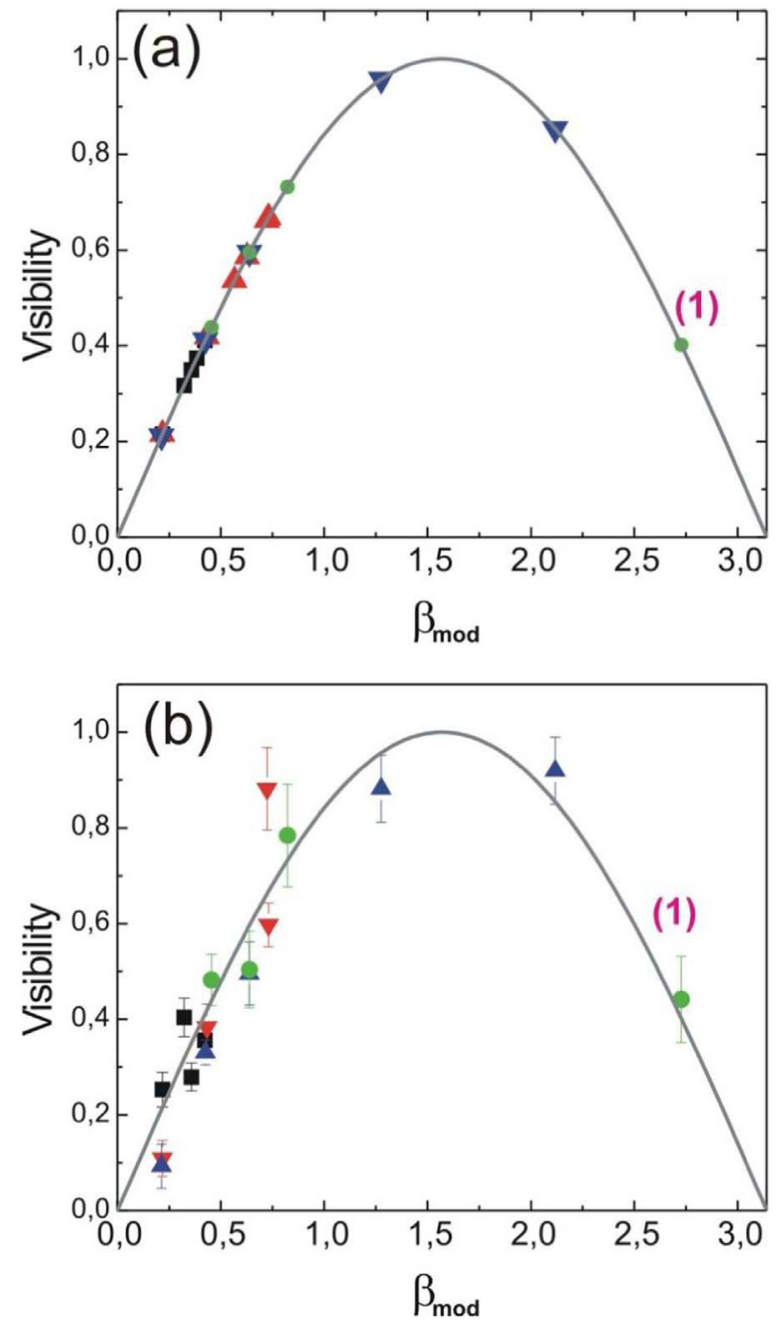

Fig. 7. Theoretical (a) and experimental (b) Lau patterns visibility vs. phase parameter $\beta_{\text {mod }}$ for: $\square L_{Z}=2,4,6$ and $10 \mathrm{~mm}$ with fixed $D=50 \mathrm{~mm}$ and $E_{0}=7 \mathrm{kV} / \mathrm{cm} ; \Delta L_{Z}=2,3,4$, 6 and $10 \mathrm{~mm}$ with fixed $D=25 \mathrm{~mm}$ and $E_{0}=7 \mathrm{kV} / \mathrm{cm} \nabla L_{Z}=2,3,4,6$ and $10 \mathrm{~mm}$ with fixed $D=3 \mathrm{~mm}$ and $E_{0}=7 \mathrm{kV} / \mathrm{cm} \bullet E_{0}=5,7$ and $9 \mathrm{kV} / \mathrm{cm}$ with fixed $D=3 \mathrm{~mm}$ and $L_{Z}=6 \mathrm{~mm} ; \bullet E_{0}=9 \mathrm{kV} / \mathrm{cm}$ with fixed $D=3 \mathrm{~mm}$ and $L_{Z}=10 \mathrm{~mm}$.

Let us observe the point indicated as (1) in both graphs. This point corresponds to $D=3 \mathrm{~mm}, L_{Z}=10 \mathrm{~mm}$ and $E_{0}=9 \mathrm{kV}$. These values of $D$ and $L_{Z}$ provide a well-defined single grating in the crystal volume. Therefore, this $E_{0}$ value guarantees the maximum index grating modulation amplitude. In this sense, the light field through this phase grating accumulates in-phase propagation producing a $\beta_{\text {mod }}=2,73>$ $>\pi / 2$ that results in a blurring of the Lau fringes reducing thereby the whole fringe contrast. Then, the visibility drops drastically, confirming thereby its sinusoidal behavior. Let us return to the discussion concerning Fig. 6c) and 6d). These results can be explained in a similar way as that argued in the point (1). The Lau fringes corresponding to $D=3 \mathrm{~mm}, E_{0}=7 \mathrm{kV}$ and $L_{Z}=10 \mathrm{~mm}$ decreases its quality with respect to $D=3 \mathrm{~mm}, E_{0}=7 \mathrm{kV}$ and $L_{Z}=6 \mathrm{~mm}$ as a consequence of that $\beta_{\bmod }>\pi / 2$ in the first case.

\section{Conclusion}

We have studied theoretically and implemented experimentally the Lau effect by using a photorefractive volume phase grating. The 3D grating is generated by imaging an incoherently illuminated Ronchi grating into a sillenite crystal. A calculation based on the OTF of an incoherent imaging system to describe the 3D intensity distribution is employed. This 3D distribution is mapped into a refractive index perturbation via the photorefractive effect. Three parameters govern the index grating modulation: the DC external electric field that controls the index modulation amplitude, the exit pupil diameter of the imaging recording system and the crystal thickness, the last two parameters related to the region in which the grating spreads out. In this way, the use of a 3D photorefractive grating in a Lau experiment turns out to control the fringes quality through the introduced parameters. A model based on a path integral formulation is used to calculate the Lau patterns' intensity generated by the mentioned volume phase grating. The Lau fringes visibility maintains the sinusoidal dependence of the planar phase grating but introducing a new proper phase parameter. Note that this parameter gathers the dependence on the output pupil diameter of the write-in process, the crystal thickness and the external applied field. In summary, an adequate combination of the three parameters which makes that $\beta_{\text {mod }}$ takes a value close to $\pi / 2$ is the best configuration to enhance the Lau image visibility.

\section{Acknowledgments}

This research was performed under the grants: CONICET No. 0849/16 and 0549/12 (Argentina) and Facultad Ingeniería, Universidad Nacional de La Plata No. 11/I215 (Argentina).

\section{References}

[1] E. Lau, Beugungserscheinungen an Doppelrastern, Ann. Phys. 6 (1948) 417-423.

[2] K. Patorski, The self-imaging phenomenon and its applications, Prog. Opt. 27 (1989) 3-108.

[3] T. Jinhong, Theoretical analysis of the Lau effect with a sinusoidal phase grating, J. Mod. Opt. 34 (1987) 307-313.

[4] J.C. Barreiro, P. Andres, J. Ojeda-Castañeda, Lau effect with only phase gratings, Opt. Commun. 73 (1989) 106-110.

[5] M. Tebaldi, L. Angel Toro, N. Bolognini, Interferometry based on Lau effect with a grating registered in a photorefractive crystal, Opt. Laser Technol. 31 (1999) $127-134$.

[6] H. Bartelt, Yajun Li, Lau interferometry with cross grating, Opt. Commun. 48 (1983) 1-6.

[7] H. Bartelt, J. Jahns, Interferometry based on the lau effect, Opt. Commun. 30 (1979) 268-274.

[8] L. Angel, M. Tebaldi, R. Henao, Phase stepping in Lau interferometry, Opt. Commun. 164 (1999) 247-255.

[9] J. Ojeda Castañeda, J. Barreiro, J. Ibarra, Schardin-Lau interferometer, Opt. Commun. 67 (1988) 325-330.

[10] M. Tebaldi, J. Rueda, N. Bolognini, Talbot interferometer based on a birefringence grating, Opt. Commun. 185 (2000) 65-76.

[11] J. Jahns, A.W. Lohmann, The Lau effect: a diffraction experiment with incoherent illumination, Opt. Commun. 28 (1979) 263-267.

[12] F. Gori, Lau effect and coherence theory, Opt. Commun. 31 (1979) 4-8.

[13] R. Sudol, B.J. Thompson, An explanation of the Lau effect based on coherence theory, Opt. Commun. 31 (1979) 105-110.

[14] T. Jinhong, The diffraction near fields and Lau effect of a square-wave modulated phase grating, J. Mod. Opt. 35 (1988) 1399-1408.

[15] M. Tebaldi, L.A. Toro, N. Bolognini, Lau fringe formation using a grating registered in a BSO crystal, Opt. Eng. 37 (1998) 2971-2978.

[16] G. Forte, A. Lencina, M. Tebaldi, N. Bolognini, Self-imaging by a volume grating, Opt. Commun. 284 (2011) 2494 (99).

[17] G. Forte, A. Lencina, M. Tebaldi, N. Bolognini, Talbot effect by a photorefractive volume phase grating, Appl. Opt. 51 (2012) 479 (85).

[18] P. Yeh, Introduction to Photorefractive Nonlinear Optics, Wiley, New York, 1993.

[19] A.T. Efremidis, N.C. Deliolanis, C. Manolikas, E.D. Vanidhis, Dispersion of electrooptic coefficients in sillenite crystal, Appl. Phys. B 95 (2009) 467 (73).

[20] M. Tebaldi, M.C. Lasprilla, N. Bolognini, Polarization-sensitive readout of selfimages, Opt. Eng. 36 (1997) 2216 (24). 